\title{
Orbit determination across unknown maneuvers using the essential thrust-Fourier-coefficients ${ }^{\text {th }}$
}

\author{
The University of Colorado at Boulder \\ 429 UCB, Boulder, CO 80309-0429 \\ Hyun Chul Ko*, Daniel J. Scheeres
}

\begin{abstract}
Any maneuver performed by a satellite transitioning between two arbitrary orbital states can be represented as an equivalent maneuver involving Thrust-Fourier-Coefficients (TFCs). With a selected TFC set as a basis, a thrust acceleration can be constructed to interpolate two unconnected states across an unknown maneuver. This representation technique with TFCs enables us to facilitate the analytical propagation of uncertainties of the satellite state. This approach allows for the usage of existing pre-maneuver orbit estimation to compute the orbit solution after the unknown maneuver. In this paper, we applied this approach to orbit determination (OD) problems across unknown maneuvers by appending different combinations of TFCs to the state vector in the batch filter. The aim is to investigate how different maneuver representations with different TFC sets affect the OD solution across unknown maneuvers. Simulation results show that each TFC set provides different representation of the unknown perturbing acceleration, which yields varying magnitudes of delta velocity for a given maneuver. However, OD solutions across unknown maneuvers using different TFC sets display equivalent performance over the post-maneuver arc as long as those TFC sets are capable of generating the apparent secular motion caused by a given unknown maneuver.
\end{abstract}

Keywords: Orbit determination, Maneuver representation, Unknown maneuver, Thrust-Fourier-Coefficients (TFCs)

\section{Introduction}

Tracking satellites and predicting their future trajectories is an important subject in space operations. The problem is to propagate the spacecraft orbit state to a future time along with a suitable estimate of its confidence boundary. When satellites perform non-announced maneuvers, post-maneuver analysis tries to reconstruct the orbit trajectory resulting from the maneuver by using orbit determination (OD) results. Without compensating for unmodeled maneuvers, the reconstructed post-maneuver orbit will have inaccurate state predictions and confidence regions. This inaccurate OD solution can be improved by modifying orbital dynamics, or using additional measurements. However, when maneuver dynamics are unknown and a limited number of measurements are available only after a maneuver,

\footnotetext{
This paper was presented at the 65th International Astronautical Congress in Toronto

* Corresponding author

Email addresses: hyun. ko@colorado.edu (Hyun Chul Ko), scheeres@colorado.edu (Daniel J. Scheeres)
} 
a pre-maneuver OD estimation must be incorporated with the post-maneuver measurements to obtain reliable OD representations and OD solutions.

\section{Unknown maneuver representation with thrust-Fourier-coefficients}

It has been shown that the TFC representation of perturbing acceleration can be used to interpolate any separate states and provide a required acceleration to generate an apparent secular behavior [7]. This unknown maneuver representation with TFCs is a mathematical model whose purpose is to rigorously represent the perturbing motion of a satellite under unknown accelerations. When a spacecraft is exposed to an unknown maneuver, the perturbing acceleration, $\vec{U}$, can be represented as a Fourier series expansion in eccentric anomaly $(E)$ along three orthogonal 
directions (radial, R; along-track, S; cross-track, W) [9] :

$$
\begin{aligned}
\vec{U} & =U_{R} \hat{\boldsymbol{r}}+U_{S} \hat{\boldsymbol{s}}+U_{W} \hat{\boldsymbol{w}} \\
U_{R} & =\sum_{k=0}^{\infty}\left[\alpha_{k}^{R} \cos k E+\beta_{k}^{R} \sin k E\right] \\
U_{S} & =\sum_{k=0}^{\infty}\left[\alpha_{k}^{S} \cos k E+\beta_{k}^{S} \sin k E\right] \\
U_{W} & =\sum_{k=0}^{\infty}\left[\alpha_{k}^{W} \cos k E+\beta_{k}^{W} \sin k E\right]
\end{aligned}
$$

in which $\alpha_{k}$ and $\beta_{k}$ are named Thrust-Fourier-Coefficients (TFCs). Substituting the Fourier series representation of perturbing acceleration components into the Gauss equations, the averaged dynamics equations were found to be a function of only 14 essential TFCs [6].

$$
\vec{c}_{14}=\left[\alpha_{0}^{R} \alpha_{1}^{R} \alpha_{2}^{R} \beta_{1}^{R} \alpha_{0}^{S} \alpha_{1}^{S} \alpha_{2}^{S} \beta_{1}^{S} \beta_{2}^{S} \alpha_{0}^{W} \alpha_{1}^{W} \alpha_{2}^{W} \beta_{1}^{W} \beta_{2}^{W}\right]^{T}
$$

With these 14 essential TFCs, the profile of any unknown thrust can be represented as follows :

$$
\begin{aligned}
\vec{U} & =\left(\alpha_{0}^{R}+\alpha_{1}^{R} \cos E+\beta_{1}^{R} \sin E+\alpha_{2}^{R} \cos 2 E\right) \hat{\boldsymbol{r}} \\
& +\left(\alpha_{0}^{S}+\alpha_{1}^{S} \cos E+\beta_{1}^{S} \sin E+\alpha_{2}^{S} \cos 2 E+\beta_{2}^{S} \sin 2 E\right) \hat{\boldsymbol{s}} \\
& +\left(\alpha_{0}^{W}+\alpha_{1}^{W} \cos E+\beta_{1}^{W} \sin E+\alpha_{2}^{W} \cos 2 E+\beta_{2}^{W} \sin 2 E\right) \hat{\boldsymbol{w}}
\end{aligned}
$$

To achieve a given orbital transfer in the 6-dimensional orbit space, several efficient sets of 6 TFCs were selected to make a fast assessment of any perturbing acceleration [7]:

$$
\begin{aligned}
\vec{c}_{e s s 1} & =\left[\begin{array}{llllll}
\alpha_{0}^{R} & \alpha_{0}^{S} & \alpha_{1}^{S} & \beta_{1}^{S} & \alpha_{1}^{W} & \beta_{1}^{W}
\end{array}\right]^{T} \\
\vec{c}_{e s s 2} & =\left[\begin{array}{llllll}
\alpha_{0}^{R} & \beta_{1}^{R} & \alpha_{0}^{S} & \beta_{1}^{S} & \alpha_{1}^{W} & \beta_{1}^{W}
\end{array}\right]^{T} \\
\vec{c}_{\text {ess } 3} & =\left[\begin{array}{llllll}
\alpha_{0}^{R} & \alpha_{1}^{R} & \alpha_{0}^{S} & \alpha_{1}^{S} & \alpha_{1}^{W} & \beta_{1}^{W}
\end{array}\right]^{T} \\
\vec{c}_{\text {ess } 4} & =\left[\begin{array}{llllll}
\alpha_{0}^{R} & \alpha_{1}^{R} & \beta_{1}^{R} & \alpha_{0}^{S} & \alpha_{1}^{W} & \beta_{1}^{W}
\end{array}\right]^{T} \\
\vec{c}_{\text {ess } 5} & =\left[\begin{array}{llllll}
\alpha_{1}^{R} & \alpha_{0}^{S} & \alpha_{1}^{S} & \beta_{1}^{S} & \alpha_{1}^{W} & \beta_{1}^{W}
\end{array}\right]^{T} \\
\vec{c}_{\text {ess } 6} & =\left[\begin{array}{llllll}
\alpha_{1}^{R} & \beta_{1}^{R} & \alpha_{0}^{S} & \beta_{1}^{S} & \alpha_{1}^{W} & \beta_{1}^{W}
\end{array}\right]^{T}
\end{aligned}
$$

Like the full set of 14 TFCs $\left(\vec{c}_{14}\right)$, these different combinations of 6 TFCs can represent unknown perturbations and provide an analytical solution of control profile to connect two separated states across unknown maneuvers. For example, an unknown perturbing acceleration can be represented by using the 6 th essential TFC set $\left(\vec{c}_{e s s 6}\right)$ :

$$
\begin{aligned}
\vec{U}= & \left(\alpha_{1}^{R} \cos E+\beta_{1}^{R} \sin E\right) \hat{\boldsymbol{r}}+\left(\alpha_{0}^{S}+\beta_{1}^{S} \sin E\right) \hat{\boldsymbol{s}} \\
& +\left(\alpha_{1}^{W} \cos E+\beta_{1}^{W} \sin E\right) \hat{\boldsymbol{w}}
\end{aligned}
$$


There are different ways to compute these TFC values for a given maneuver [7], and those computed TFC values will be different for each TFC set. However, all of them will generate the same secular behavior for a given unknown maneuver as long as they tie two separate states together.

\section{Orbit determination across unknown maneuvers}

This section briefly explains how the modified batch filter with the essential TFCs works to connect the postmaneuver tracking data to the pre-maneuver orbit state. Without using the pre-maneuver orbit information, achieving reliable post-maneuver state estimation given a small number of post-maneuver measurements is a difficult problem. A conventional method used at the time of encountering an unknown maneuver is to initiate the batch filter over a post-maneuver tracking arc with large initial uncertainties [10]. To distinguish this batch filter from the modified batch filter in this paper, we label it as a regular batch filter that estimates only post-maneuver satellite position and velocity vectors. With the regular batch filter, the state covariance matrix is inflated to address the uncertainty in the post-maneuver estimation and the accuracy of state estimate is low at this stage. Making use of the pre-maneuver OD solution through the TFC maneuver representation will improve the overall OD solutions, specially when there is no measurement available during the maneuvering period.

Before utilizing the TFC representation to maintain OD across unknown maneuvers, it is necessary to examine how the covariance propagation changes with different maneuver dynamics connecting two separate states. Previously, Ko and Scheeres developed an analytic expression for the solution of state difference between the true and the represented trajectories, and proved that the covariance propagation in the linear region is independent of the dynamics as long as they represent the same orbital change due to unknown perturbations [8] :

$$
\begin{aligned}
P_{T}\left(t_{f}\right) & =\Phi_{T}\left(t_{f}, t_{0}\right) P_{0} \Phi_{T}\left(t_{f}, t_{0}\right)^{T} \\
& =\Phi_{R}\left(t_{f}, t_{0}\right) P_{0} \Phi_{R}\left(t_{f}, t_{0}\right)^{T}+H . O . T \\
& \simeq \Phi_{R}\left(t_{f}, t_{0}\right) P_{0} \Phi_{R}\left(t_{f}, t_{0}\right)^{T}=P_{R}\left(t_{f}\right)
\end{aligned}
$$

in which $P$ is the covariance matrix and $\Phi\left(t_{f}, t_{0}\right)$ is the state transition matrix (STM) that maps deviations in the initial state from the pre-maneuver time $\left(t_{0}\right)$ to the post-maneuver time $\left(t_{f}\right)$ using either the true dynamics or the represented dynamics. The subscripts $T$ and $R$ designate their related true and represented dynamics respectively. H.O.T means the high order terms that is neglected in the linear region. This fundamental finding served as the baseline of this paper and was applied to orbit determination problems using the representation technique with different TFC sets in the batch filter.

Modifying the regular batch filter is easily done by simply augmenting the state vector with TFCs. The augmented state vector consists of time-dependent satellite position and velocity vectors and constant TFCs. By representing an 
unknown maneuver with an essential TFC set, the governing equations of motion can be rewritten as follows :

$$
\begin{gathered}
\dot{\vec{X}}(t)=\vec{F}(\vec{X}, t)+B \cdot \vec{U}(\vec{X}, t) \\
\frac{d}{d t}\left[\begin{array}{c}
\vec{r} \\
\vec{v} \\
\vec{c}_{\text {ess }}
\end{array}\right]=\left[\begin{array}{c}
\vec{v} \\
\dot{\vec{v}} \\
0_{6 \times 1}
\end{array}\right]+\left[\begin{array}{c}
0_{3 \times 3} \\
I_{3 \times 3} \\
0_{6 \times 3}
\end{array}\right] \cdot \vec{U}(\vec{X}, t)
\end{gathered}
$$

in which $\vec{F}(\vec{X}, t)$ is the known dynamics and $\vec{U}(\vec{X}, t)$ is the represented unknown acceleration. Along with the augmented state and governing dynamics equations, the state Jacobian and measurement Jacobian matrices have to be modified to complete the batch algorithm with TFCs [11] :

$$
\begin{aligned}
\frac{d \Phi_{R}\left(t, t_{0}\right)}{d t} & =\frac{\partial}{\partial \vec{X}_{0}}[\vec{F}(\vec{X}, t)+B \cdot \vec{U}(\vec{X}, t)] \\
= & \frac{\partial \vec{F}(\vec{X}, t)}{\partial \vec{X}} \cdot \frac{\partial \vec{X}(t)}{\partial \vec{X}_{0}}+B \cdot \frac{\partial U(\vec{X}, t)}{\partial \vec{X}} \cdot \frac{\partial \vec{X}(t)}{\partial \vec{X}_{0}} \\
= & {\left[\frac{\partial \vec{F}(\vec{X}, t)}{\partial \vec{X}}+B \cdot \frac{\partial U(\vec{X}, t)}{\partial \vec{X}}\right] \cdot \Phi_{R}\left(t, t_{0}\right) } \\
H & =\tilde{H} \cdot \Phi_{R}\left(t, t_{0}\right)=\frac{\partial G(\vec{X}, t)}{\partial \vec{X}} \cdot \Phi_{R}\left(t, t_{0}\right)
\end{aligned}
$$

in which $G(\vec{X}, t)$ is the measurement equation. This modification in the batch filter allows for periods of an unknown maneuver firing to be fit through without having a precise model of the event. This modified batch filter estimates the essential TFCs as well as an initial orbit state by processing a post-maneuver tracking data. It provides the least square solution that minimizes the square of the difference between the modeled observation and the actual observation. A detailed flow chart for the modified batch processor with TFCs can be found in [11] .

\section{Simulation and results}

In order to verify the performance of the modified batch filter with different TFC sets, several unknown maneuver cases are simulated and OD is performed with simulated tracking data. All simulations are performed on a low-earth orbiting (LEO) satellite and the pre-maneuver orbit state is shown in Table 1. Two-body dynamics under the uniform

Table 1: Initial orbit state of LEO satellite

\begin{tabular}{cccccc}
\hline \hline$h_{0}(\mathrm{~km})$ & $e$ & $i(\mathrm{deg})$ & $\Omega(\mathrm{deg})$ & $\omega(\mathrm{deg})$ & $\nu(\mathrm{deg})$ \\
\hline 1122 & 0.1 & 20 & 20 & 20 & 0 \\
\hline \hline
\end{tabular}

$h_{0}:$ altitude at perigee; Earth Radius : $6378.137 \mathrm{~km}$

gravity field is assumed to be the only known dynamics upon a satellite, which allows us to compare OD solution of the modified filter in a simple way. In this paper, 4 different cases of unknown maneuvers are analyzed with the modified 
Table 2: Different unknown maneuver cases

\begin{tabular}{ccc}
\hline \hline case & direction & $\Delta \mathrm{V}(\mathrm{m} / \mathrm{s})$ \\
\hline 1 & Radial (R) & $10(\mathrm{R})$ \\
2 & Along-track (S) & $10(\mathrm{~S})$ \\
3 & Cross-track (W) & $10(\mathrm{~W})$ \\
4 & Random & $6(\mathrm{R}) 9(\mathrm{~S}) 3(\mathrm{~W})$ \\
\hline \hline
\end{tabular}

filter, as is shown in Table 2. Cases 1-3 describe cases with an unknown acceleration acting on a single direction, while case 4 represents an example with a perturbation along a random direction. For all these cases, perturbing accelerations last for 1000 seconds and the actual magnitude of perturbing acceleration is $1 \times 10^{-2} \mathrm{~m} / \mathrm{s}^{2}$ for case 1-3 and $3 \sim 6 \times 10^{-3} \mathrm{~m} / \mathrm{s}^{2}$ for case 4 . The observation gap across an unknown maneuver is 1 -orbital period ( $\simeq 1.8$ hours) for all the cases and there is no measurement available during the maneuvering period. As a part of simulation, a measurement generating function produces a small number of post-maneuver measurements (range and range-rate measurements from a single ground station) that are collected at every minute after unknown maneuvers. For an initial condition, a pre-maneuver OD solution is obtained by processing a simulated pre-maneuver tracking data for 3 orbital periods, which provides an OD result with spherical uncertainty of $1 \mathrm{~m}$ in position and $1 \times 10^{-2} \mathrm{~m} / \mathrm{s}$ in velocity. Given this pre-maneuver OD solution and a set of post-maneuver measurements, the modified batch filter with each different TFC set determines a post-maneuver orbit as well as TFC values.

Simulation results are illustrated by two graphical presentations : one that compares the recovered control profile with each TFC set versus the true perturbation; the other that compares OD solutions from the modified filter to those from the regular batch filter. Simulation results and figures are very similar for different cases, so only the resulting figures for case 4, the most representative case, are shown in this paper to avoid repetition. Figures. 1 shows the represented maneuver acceleration for case 4. In this figure, the first subfigure displays estimated TFC values from the modified batch filter with 7 different TFC sets including the full set of 14 TFC. Since these 7 TFC sets have different configuration of TFCs on each direction (radial, along-track, cross-track), their estimated values are different from each other. It is noticeable that some TFCs are more dominant than others, depending on the true perturbing direction. For case 4, the radial and along-track components of TFC sets are more dominant than cross-track ones.

The second subfigure, Fig. 1(b), compares the reconstructed perturbing acceleration to the true perturbation. The shapes of the recovered control profile using 7 different TFC sets are different from each other as well as the true perturbing acceleration. However, it is shown that the magnitudes of reconstructed accelerations are found to be the same order of magnitudes as the true perturbations. This can be also shown by computing a control effort ( $\Delta \mathrm{V})$ by integrating the thrust acceleration profile over the time interval, which is shown in Table. 3. Even though the computed $\Delta$ Vs do not exactly match with true values, it still can be used to bound the control effort necessary for linking two separate states across an unknown maneuver. Note that using the full set of 14 TFCs does not exhibit any superior 


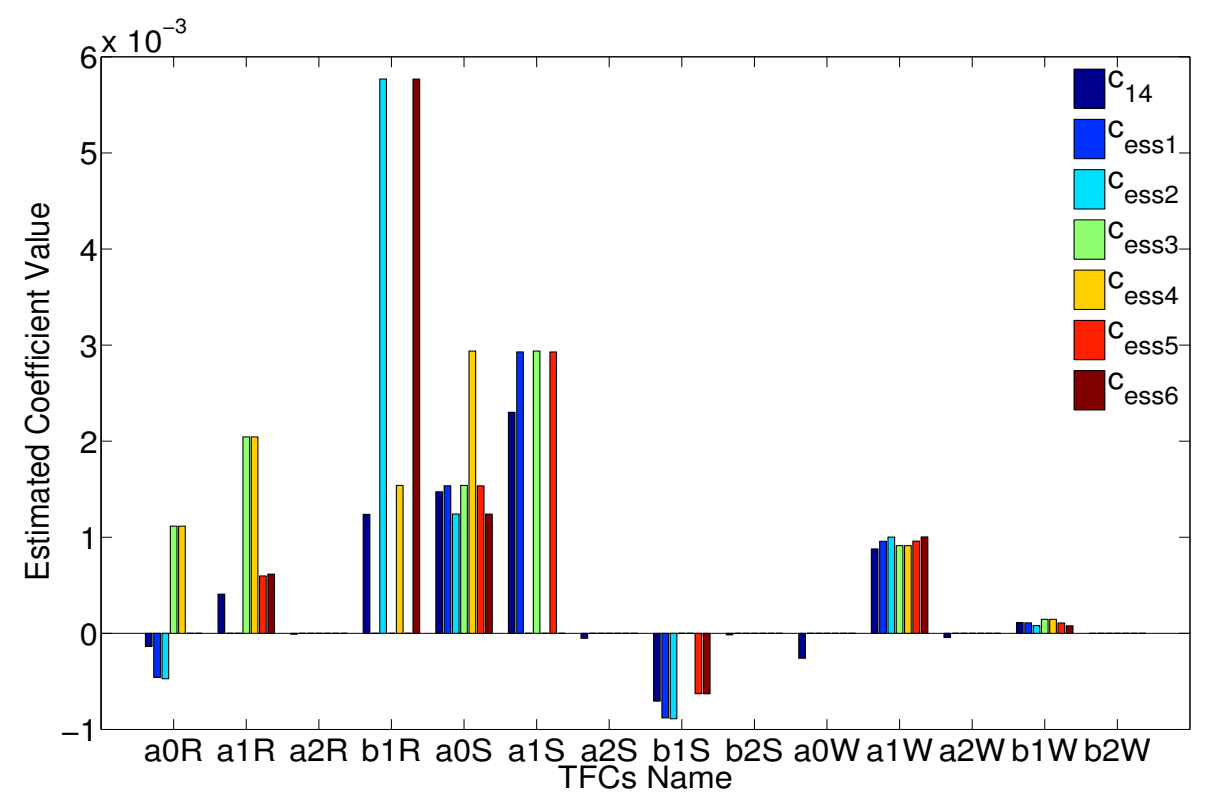

(a) Estimated TFC values for different TFC sets
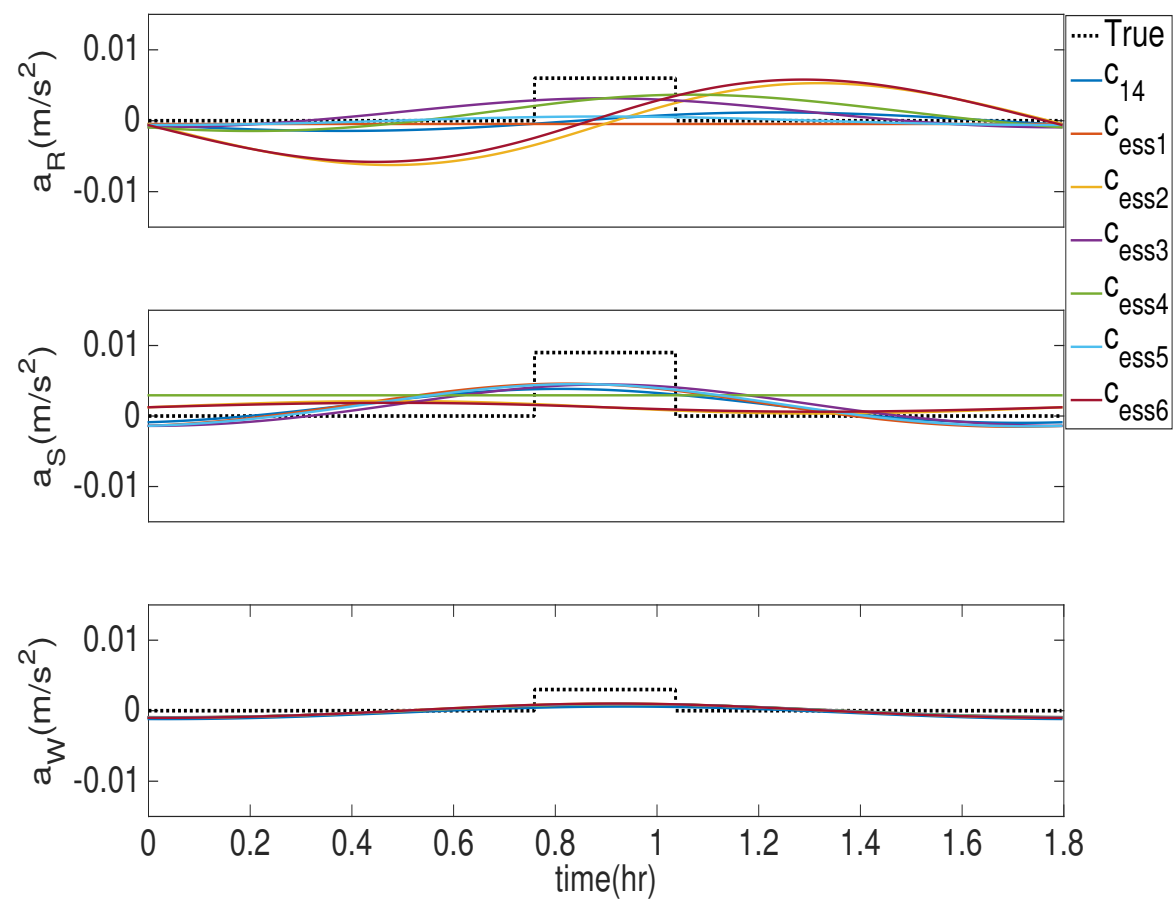

(b) Represented vs True acceleration components

Figure 1: Maneuver representation for case 4 
Table 3: True vs Estimated $\Delta \mathrm{V}$ for different cases $(\mathrm{m} / \mathrm{s})$

\begin{tabular}{cc|cccccccc}
\hline \hline & & True & $14-$ TFC set & set 1 & set 2 & set 3 & set 4 & set 5 & set 6 \\
\hline & $\mathrm{R}$ & 10 & 2.8897 & 4.7517 & 4.7497 & 15.1622 & 15.1622 & 3.9380 & 3.9677 \\
case 1 & $\mathrm{S}$ & 0 & 4.7950 & 5.9447 & 5.9401 & 0.3199 & 0.5045 & 4.2801 & 4.2744 \\
& $\mathrm{~W}$ & 0 & 0.6268 & 0.7949 & 0.7966 & 0.7502 & 0.7502 & 0.8015 & 0.8033 \\
\hline & $\mathrm{R}$ & 0 & 5.6536 & 0.3328 & 26.2376 & 0.7409 & 7.0409 & 0.2760 & 26.2400 \\
case 2 & $\mathrm{S}$ & 10 & 12.2043 & 14.5362 & 8.8852 & 14.5363 & 20.9269 & 14.5340 & 8.8843 \\
& $\mathrm{~W}$ & 0 & 0.9207 & 0.9505 & 1.1084 & -0.0673 & 0.9410 & 0.9511 & 1.1093 \\
\hline & $\mathrm{R}$ & 0 & 0.1812 & 0.1904 & 0.8080 & 0.4190 & 0.4190 & 0.1578 & 0.8143 \\
case 3 & $\mathrm{S}$ & 0 & 0.3369 & 0.4133 & 0.0906 & 0.4022 & 0.6333 & 0.4324 & 0.1556 \\
& $\mathrm{~W}$ & 10 & 10.3769 & 10.6291 & 10.6351 & 10.6360 & 10.6360 & 10.6292 & 10.6352 \\
\hline & $\mathrm{R}$ & 6 & 5.4243 & 2.9631 & 23.8513 & 9.2573 & 11.1981 & 2.4569 & 23.9105 \\
case 4 & $\mathrm{S}$ & 9 & 11.3491 & 13.6142 & 8.0233 & 13.1765 & 19.0002 & 13.3777 & 8.0180 \\
& $\mathrm{~W}$ & 3 & 3.8708 & 3.9644 & 4.1319 & 3.8002 & 3.8002 & 3.9730 & 4.1407 \\
\hline \hline
\end{tabular}

performance over other sets with 6 TFCs. In fact, the modified batch filter with the 14-TFC set does not produce more accurate OD estimation than others either, which is shown in Fig. 2-3. Also, none of those 6 TFC sets recovers the true perturbation more accurately than others, considering the control shape and the expenditure $(\Delta V)$. Therefore, we can represent an unknown maneuver with any TFC set, which still allows us to quantify the magnitude of an actual perturbation and to choose a preferred control figuration for a given maneuver.

To examine the filter performance with different TFC sets, OD solutions from the modified batch filter over onehour post-maneuver tracking arc are compared. With an updated initial state and estimated TFC values from the modified filter, the pre-maneuver orbit is propagated through an unknown maneuver period using the represented dynamics with different TFC sets. For a comparison, state errors and covariance envelopes from the regular batch filter are also computed by applying the filter over the post-maneuver tracking arc with an inflated covariance matrix. All the results on various cases are consistent with the others and simulation results of case 4 are shown in Fig. 2. Each subplot describes the errors of the state estimate from the modified filter with the 14-TFC set as $\bigcirc$, those from the modified filter with each set of 6 essential TFCs as + , and ones with the regular batch filter without TFCs as $\square$. Different covariance boundaries obtained with those filters are shown with their respective colors (black for regular batch filter; blue for batch with 14 TFCs; others for batch with 6 TFCs). Figure. 2 shows that all post-maneuver state errors are within their uncertainty boundaries and they are similar to each other. The modified filter with different TFC sets is able to deliver a valid post-maneuver OD solution across unknown maneuvers. However, this figure does not reveal the advantage of using the modified batch filter with essential TFCs over the regular batch filter.

To investigate the benefit of applying the TFC representation approach, the accuracy of OD solutions is examined 


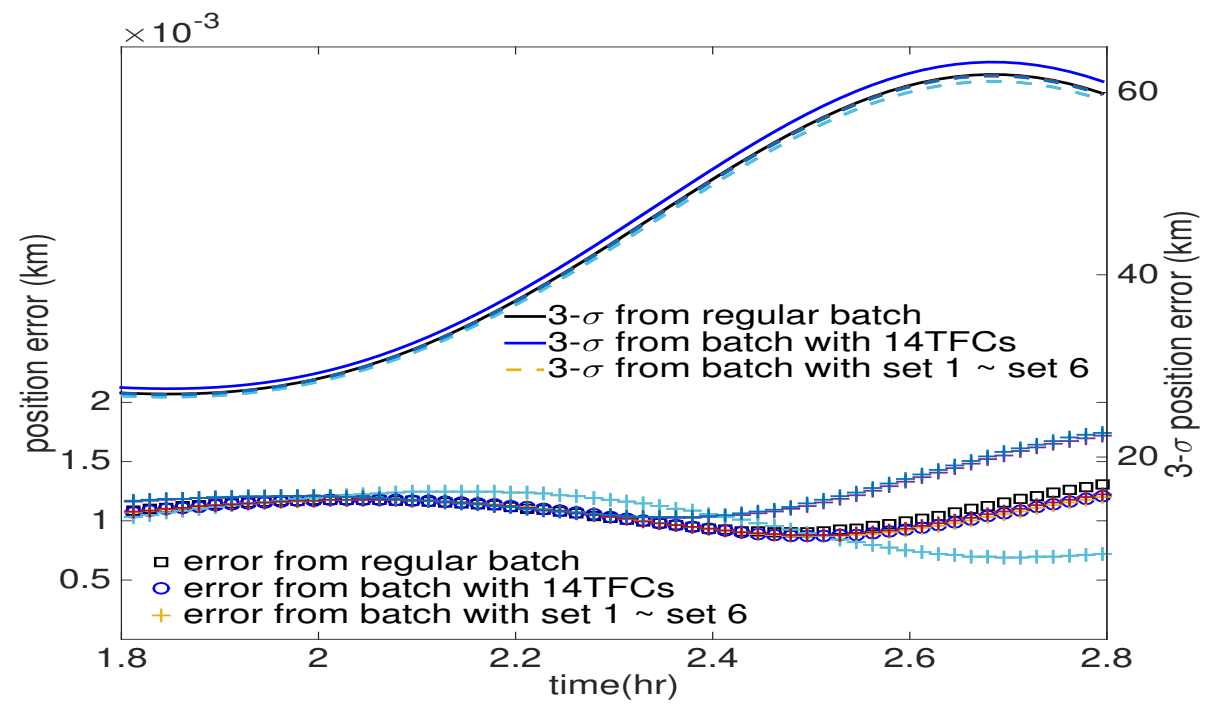

(a) Position error with $3 \sigma$ uncertainty boundary

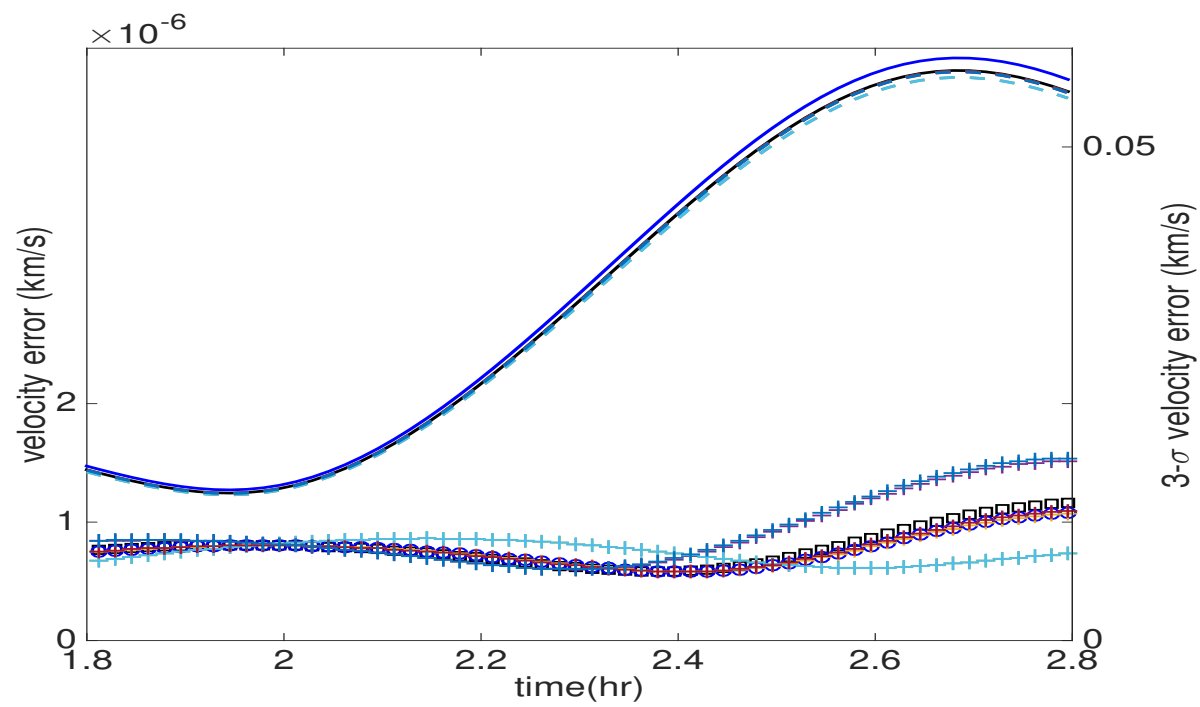

(b) Velocity error with $3 \sigma$ uncertainty boundary

Figure 2: Post-maneuver OD solution from different batch filters for case 4 with one hour tracking data 
with different size of post-maneuver tracking data duration $(1 \sim 90$ measurements in $1 \sim 90$ minutes $)$. When a new measurement data comes in, the modified batch filter with different TFC sets as well as the regular batch filter are reinitiated with a new measurement set and estimate post-maneuver orbit solutions. Figure. 3 displays a collection of

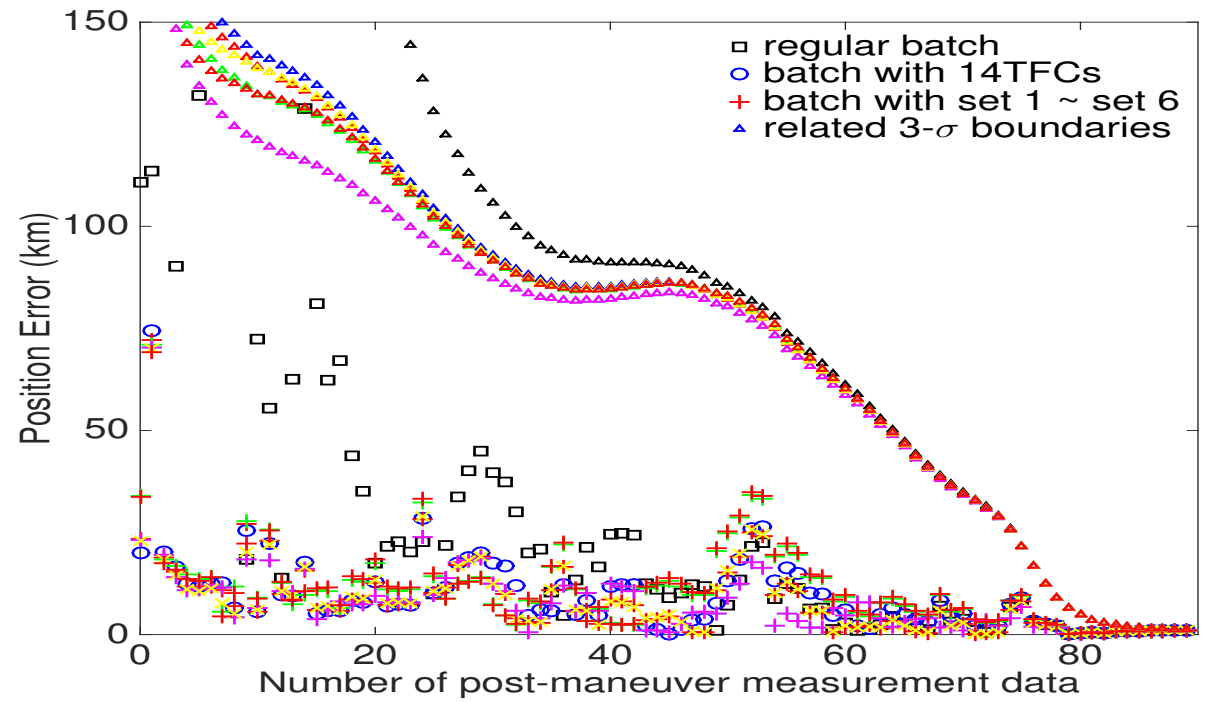

(a) Position error with $3 \sigma$ uncertainty boundary

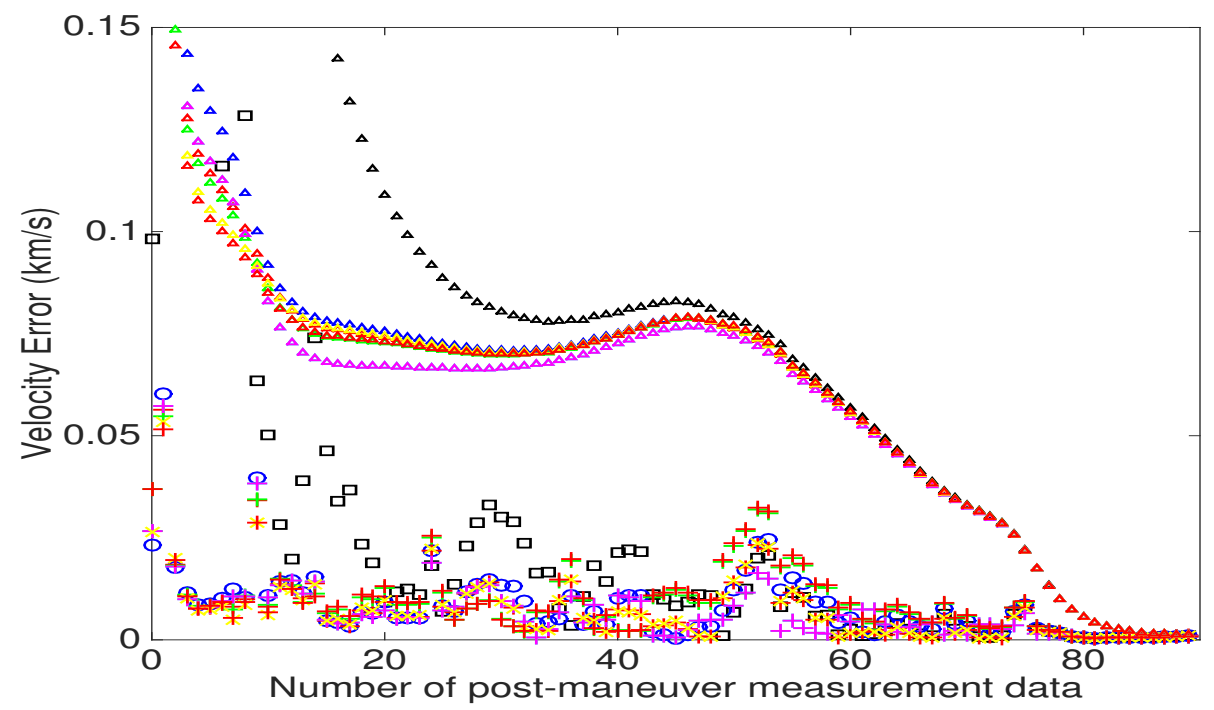

(b) Velocity error with $3 \sigma$ uncertainty boundary

Figure 3: OD solution with different sizes of tracking data for case 4

OD solutions estimated with different sizes of tracking data duration. State errors and their uncertainty boundaries are computed at their respective last measurement time of each tracking duration. Both subfigures show that state errors are well within their 3- $\sigma$ uncertainty boundaries and each OD solution from the modified batch filter with different TFC sets is consistent with each other regardless of a size of measurement data. This validates that the covariance 
propagation in the linear region is independent of the represented dynamics, by showing that different maneuver representations using different TFC sets produce similar OD solutions across unknown maneuvers. It is also found in post-maneuver state and uncertainty estimation.

\section{Acknowledgment}

The authors acknowledge support through Air Force Office of Scientific Research grant FA9550-11-1-0188.

\section{References}

[4] M. J. Holzinger, D. J. Scheeres, K. T. Alfriend, Object correlation, maneuver detection, and characterization using control distance metrics, Journal of Guidance, Control, and Dynamics 35 (4) (2012) 1312-1325. 
[5] D. P. Lubey, D. J. Scheeres, Identifying and quantifying mismodeled dynamics via optimal control problemidentifying and quantifying mismodeled dynamics via optimal control problem distance metrics, no. AIAA 2012-5020 doi: 10.2514/6.2012-5020, The 2012 AIAA/AAS Astrodynamics Specialists Meeting, Minneapolis, MN, 2012.

[6] J. S. Hudson, D. J. Scheeres, Reduction of low-thrust continuous controls for trajectory dynamics, Journal of Guidance, Control, and Dynamics 32 (3) (2009) 780-787.

[7] H. C. Ko, D. J. Scheeres, Essential thrust-fourier-coefficient set of averaged gauss equations for orbital mechanics, Journal of Guidance, Control, and Dynamics 37 (4) (2014) 1236-1249.

[8] H. C. Ko, D. J. Scheeres, Event representation based orbit determination across unknown space events, Journal of Guidance, Control, and Dynamics (2015) doi: http://arc.aiaa.org/doi/abs/10.2514/1.G001050.

[9] R. Haberman, Applied Partial Differential Equations with Fourier Series and Boundary Value Problems, 4th Edition, Pearson Prentice-Hall, Upper Saddle River, NJ, 2004.

[10] T. Johnson, Tdrs-3 orbit determination across unknown maneuvers, Tech. rep., 4th International Conference on Astrodynamic Tools and Techniques, Madrid Spain (May 2010).

[11] H. C. Ko, D. J. Scheeres, Spacecraft orbit anomaly representation using thrust-fourier-coefficients with orbit determination toolbox, Vol. 1, Proceedings of the Advanced Maui Optical and Space Surveillance Technologies Conference, Maui, Hawaii, 2014, p. 63. 